\title{
La Industria de la Investigación como instancia mediadora de la producción de conocimiento sociológico
}

\author{
Research Industry as a mediating instance in the production of sociological \\ knowledge
}

Coral HERNÁNDEZ FERNÁNDEZ

Universidad Complutense de Madrid - España

chernandezf@pdi.ucm.es

Cómo citar este artículo: HeRnándeZ FeRnÁNDEZ, Coral (2015): «La Industria de la Investigación como instancia mediadora de la producción de conocimiento sociológico», Mediaciones Sociales, no 14, pp. 129-142. DOI: http://dx.doi.org/10.5209/rev_MESO.2015.n14.51562

Recibido: 9 de octubre de 2015.

Aceptado: 30 de noviembre de 2015.

\section{RESUMEN}

Este artículo toma como punto de partida la investigación realizada para la tesis doctoral "La producción de conocimiento sociológico en España”, en la que se analiza el papel que tuvo -y aún sigue teniendo- el sector empresarial dedicado a la producción de investigación sobre el consumo en el desarrollo de la sociología aplicada en nuestro país. Se estudian las características de dicha Industria de la Investigación, como institución mediadora del conocimiento sociológico, que participa en las tareas de control social, como modelo de orden. El artículo evidencia cómo tanto la Industria de la Investigación como sus modos de producción y sus productos finales pueden -y deben- ser analizadas e interpretadas desde la teoría de la mediación social y la producción social de conocimiento.

PALABRAS CLAVE: Industria de la Investigación, institución mediadora, investigación social aplicada, producción de conocimiento, sociología del conocimiento. 


\begin{abstract}
This paper is based on the doctoral thesis research "The production of sociological knowledge in Spain", which analysis the past and current role played by the industry of knowledge production about consumption in the development of applied sociology in Spain. The paper studies the features of this Research Industry, as a mediating institution of the sociological knowledge, which is involved in social control tasks, as an order model. The article makes clear how the Research Industry as well as its modes of production and its final products can be -and should be- analysed and interpreted from the social mediation theory and from the social production of knowledge.
\end{abstract}

KEYWORDS: Research Industry, mediating institution, applied social research, knowledge production, knowledge sociology.

Sumario: 1. El contexto social de los orígenes de la sociología aplicada en España. 2. El tránsito a la sociedad de consumo y la creciente necesidad de una investigación social aplicada. 3. El despertar del interés institucional por la investigación social y los estudios sociológicos. 4. La consolidación de la Industria de la Investigación. 5. La Industria de la Investigación como institución mediadora. 6. Conclusión: el papel instrumental de la industria de la investigación en la reproducción y el control social. Bibliografía.

\title{
1. EL CONTEXTO SOCIAL DE LOS ORÍGENES DE LA SOCIOLOGÍA APLICADA EN ESPAÑA
}

La investigación social aplicada es una actividad investigadora cuya finalidad es la de crear modelos praxeológicos de la realidad, que son a la vez analíticos ya que mediante el análisis de los hechos, tratan de comprender y hacer comprender la realidad; predictivos puesto que, a partir de la comprensión de los hechos, tratan de predecir las alternativas posibles de realidad futura y operacionales dado que la información que producen tiene el objetivo de actuar sobre la realidad social para cambiarla ${ }^{1}$.

Este campo de la sociología aplicada, en nuestro país, se ha desarrollado históricamente en paralelo al desarrollo de la sociedad de consumo, de forma que ha existido -y sigue existiendo, al menos en parte- una trayectoria común de la investigación social y la investigación comercial en muchos momentos de nuestra historia reciente.

1 Según describe Martín Serrano (1978: 19), "los modelos teóricos que son isomorfos de los modelos funcionales son los únicos que se consideran satisfactorios desde el punto de vista del conocimiento del sistema y del control sobre el sistema. Esta clase de modelos, a la vez teóricos y funcionales, son analíticos, predictivos y operacionales, es decir, son modelos praxeológicos. La aceptación de la hipótesis del isomorfismo entre código funcional del sistema y código del modelo teórico del sistema significa que la misma lógica se considera válida para explicar las prácticas y el raciocinio, los procesos reales y los procesos cognitivos. (...) Traducida en el lenguaje de las ciencias sociales, significa que el sistema de la acción y el sistema de las representaciones se consideran lógicamente equiparables, en cuanto que la representación es una forma de acción especializada en organizar el sistema". 
De hecho, la investigación social de tipo comercial se inició en España antes que la de orientación sociológica, debido a la influencia de las primeras multinacionales que llegaron a España tras la firma de los acuerdos bilaterales con Estados Unidos en 1953. Estas compañías trajeron consigo un modelo empresarial que en el que la investigación del consumo y del consumidor formaban parte integrante de su sistema de gestión. Sin embargo, en España había una acusada carencia de información institucional de tipo sociológico, por lo que los estudios de consumo tuvieron que adentrarse en el terreno de la investigación social, aunque fuera con fines meramente demográficos, para realizar una sociología "contable" (quienes, cuantos, dónde...) que permitiera, simplemente, conocer la realidad social en la que sus mercados iban a crecer y desarrollarse.

Manuel Martín Serrano, que vivió en primera persona ese primer desarrollo de la sociología aplicada en nuestro país a partir de mediados de la década de 1950, habla de una deliberada intención institucional de evitar el desarrollo real del pensamiento sociológico, que quedaba limitado o bien a la orientación "teológico-filosófica" o bien a los aspectos puramente demográficos:

"En esos primeros años, hay un interés específico en que dicho conocimiento sociológico se circunscriba al ámbito económico-demográfico, o al meramente filosófico. El Instituto Balmes domina la producción de conocimiento y aunque se registran varios intentos desde otras aproximaciones teóricas no religiosas, estos son sistemáticamente frustrados desde las instancias oficiales.

Se para cualquier intento de enseñanza vinculada a la sociología, con el sabio argumento de que, cuando en la Universidad Complutense hubo un desarrollo sociológico, se produjo el desastre que llevó a los intelectuales hacia la república. Aquí hubo una barrida completa, después de la guerra civil, de los profesores que habían trabajado para las Ciencias Sociales en clave de transformación sociopolítica y con los criterios de la Institución Libre de Enseñanza. Eso fue interesantísimo, lo más interesante que ha habido en este país como propuesta de transformación sociopolítica. Y lo que sucedió es que lo laminaron, porque todos ellos tuvieron que irse a la emigración" (entrevista a Manuel Martín Serrano, julio de 2011).

\section{EL TRÁNSITO A LA SOCIEDAD DE CONSUMO Y LA CRECIENTE NECESIDAD DE UNA INVESTIGACIÓN SO- CIAL APLICADA}

En la década de 1960 España comenzó a dar sus primeros pasos hacia la sociedad de consumo, imponiendo a las empresas una creciente necesidad de información social orientada al mercado. La producción de este tipo de conocimiento fue relativamente bien acogido por el régimen franquista, que no solo toleraba sino que incluso favorecía el desarrollo de la investigación comercial, mientras que seguía limitando y entorpeciendo la sociológica. 
La actividad investigadora de las empresas y su necesidad de conocer la estructura y características del consumo en España dio alas al desarrollo de la investigación social aplicada. Por un lado, porque muchos de aquellos estudios iniciales requerían que el análisis se hiciese desde un punto de vista sociológico; por otro, porque muchos psicólogos sociales y sociólogos habían encontrado en los estudios de consumo un campo en el que desarrollar una actividad de investigación para el conocimiento de la sociedad que, por las condiciones sociopolíticas del país, hubiera sido muy difícil de lograr en otros ámbitos.

“Ellos [las compañías multinacionales y las agencias de publicidad] trajeron a sus propios técnicos, para estudiar el mercado español, Pero la necesidad de poner en marcha los equipos de los institutos de investigación en nuestro país, todavía agrario y en un acelerado proceso de transformación en una sociedad de servicios e industrial- transformación que se completaría en menos de una década- produce una demanda extraordinaria de profesionales de los estudios de mercado. Te estoy hablando desde el año 58 hasta el año 64-65. Se pagaba muy bien y había trabajo para todo el que lo supiera y quisiera hacer, y esas posibilidades ocupan en primer lugar a los profesionales que habían hecho economía, pero lo que pasa es que para hacer análisis en clave de consumo hace falta introducir variables de comportamiento, de actitudes, de necesidades y de percepción, con lo que el desarrollo de los estudios de mercado arrastra a psicólogos sociales y a los pocos sociólogos que había en aquel momento, a trabajar en temas sociológicos vinculados al consumo y por ahí es por donde entra de verdad la sociología, que tiene una influencia importante en la transformación del país. Y está en las empresas, porque ese nivel no se puede subestimar, porque es un momento en el que está cambiando el país y las condiciones de vida.

Todas estas personas que entraban en empresas multinacionales tenían su propio lavado de coco. Venían por aquí equipos que les contaban cómo tenían que proceder con esto del marketing, que no se sabía entonces muy bien lo que era, pero detrás de la intervención del marketing había planificación, análisis de cómo se comportaba la población, del mercado, etc. Y esa línea instrumental o práctica, como la quieras llamar, ha sido tan importante en este país como la sociología, porque el lado sociológico al final de los 60 tiene un enorme peso no solo sociopolítico, sino también de poder, de quienes vinieron a hacer estudios sociales financiados por la administración" (entrevista a Manuel Martín Serrano, julio de 2011).

De este modo, en la investigación social de tipo comercial acabaron encontrando refugio intelectual y campo de experimentación muchos de quienes posteriormente serían figuras destacadas en el desarrollo de la sociología académica, como pudieron ser los casos de Jesús Ibáñez, Ángel de Lucas o Alfonso Ortí.

"La forzada trayectoria profesional de Jesús Ibáñez resulta paradigmática en muchos aspectos. (...) Tras su proceso y encarcelamiento en 1956, y consecuente exclusión entonces de la esfera pública, (...) Ibáñez tuvo que encontrar un modus vivendi fuera de la Universidad y (...) de la Administración Pública. Lo encontró, en el nuevo sector de la 
«investigación de mercados» -en aquel momento en su fase inicial-, siendo contratado como Director técnico del Gabinete de estudios o Instituto Eco, en el momento mismo de su fundación, en 1958. (...) El repliegue y concentración de Ibáñez en el «trabajo sociológico empírico» (...), iba a dar lugar a una original renovación metodológica (...) de la «sociología del consumo» (...).

Desde principios de los años 1960, un primer grupo de colaboradores y discípulos (...) carentes todos de una plataforma institucional democrática (...) para desarrollar una (...) investigación social «crítica» (...) y enmudecidos también en la esfera pública (...), encontramos en el "trabajo sociológico concreto», en torno a las transformaciones del consumo y a las estrategias mercadológicas, una vía de profesionalización «vicaria» y un «banco de pruebas» metodológico, en espera de mejores tiempos" (Ortí, 2007: 42-44).

Como relata Martín Serrano, quienes trabajaban en investigación de mercados en aquel momento fueron testigos del reacomodo social que se produjo durante toda la década, que tuvo lugar en primer lugar como modelo transmitido a través de la televisión y que, en aquel contexto, resultaba totalmente revolucionario.

"Hubo una explosión de publicidad y hacer publicidad era crear un imaginario en los anuncios, coincidiendo -y no era casualidad que fuera tan rápido- con la entrada de la televisión en los hogares. Esos imaginarios crean otra sociedad que fue antes en la televisión de que fuese en la vida real. Quienes estudiábamos las motivaciones que tenían las personas para consumir, estábamos asistiendo a un reacomodo de las identidades, de los objetivos y de las formas de vida que resultaba fascinante, y que pasó en nada de tiempo. No se puede imaginar cómo ese volumen de personas que dejan los espacios rurales y que se vienen a la ciudad a trabajar, a vivir en las ciudades dormitorio, no solo cambian de sitio, sino que tienen que cambiar todas esas otras cosas, y esa fue la mayor revolución que se vivió en este país. Y pasó durante el franquismo y pese al franquismo, pero también de alguna manera, pasó porque (las autoridades) estaban en otra historia, y no tuvieron la suficiente perspectiva para darse cuenta de que el consumo, en ese contexto, era revolucionario" (entrevista a Manuel Martín Serrano, julio de 2011).

Este predominio de la investigación sobre el consumo y los consumidores, desarrollada de acuerdo a los intereses comerciales de las empresas, trajo consigo el temprano desarrollo de un segmento empresarial especializado, que concentró en manos privadas la mayor parte de la infraestructura productiva necesaria para la investigación social aplicada.

La actividad productiva de este sector empresarial, casi desde sus inicios estuvo organizada en torno a un código ético y de procedimiento común a todas las empresas, que en 1968 se vio oficializado a través de la Asociación Española de Estudios de Mercado y Opinión (AEDEMO), creada por la acción conjunta de representantes de los anunciantes, las agencias de publicidad y los institutos de investigación y cuyo principal objetivo, según se expresa en sus estatutos (todavía vigentes), era "la difusión y control de las técnicas empleadas en la Investigación Comercial". 


\section{EL DESPERTAR DEL INTERÉS INSTITUCIONAL POR LA INVESTIGACIÓN SOCIAL Y LOS ESTUDIOS SOCIOLÓGICOS}

A mediados de la década de 1960, cuando España daba sus primeros pasos en la sociedad de consumo, se produjeron una serie de importantes cambios sociales que dieron lugar a un creciente interés por la investigación sociológica aplicada y por los métodos y las técnicas de investigación. La necesidad de conocer de primera mano la reestructuración que estaba sufriendo la sociedad española dio lugar a un "despertar" del interés institucional por el conocimiento sociológico, que se plasmó en la creación, en 1963, del Instituto de Opinión Pública (IOP) y de la Revista Española de Opinión Pública, que resultarían trascendentales en la definitiva institucionalización de la sociología, proporcionando tanto una base para la investigación empírica como un importante medio de difusión (Lamo de Espinosa, 1992).

Manuel Martín Serrano cuenta sus impresiones sobre el Instituto y el papel que jugó en el desarrollo del enfoque sociológico, resaltando la importancia que tuvo la incorporación al mismo de profesores e investigadores procedentes de la Facultad de Ciencias Políticas, a los que se unían los recién llegados de Estados Unidos, que traían una mentalidad tecnocrática y positivista, muy diferente de las posiciones éticofilosóficas que habían dominado la sociología española hasta ese momento:

"El gran impacto lo produjo, creando las condiciones para el despegue de la sociología, Manuel Fraga Iribarne, cuando creó el Instituto de la Opinión Pública, En primer lugar, porque supuso que «la opinión» fuese un objeto de estudio que iba a tener un lugar en el régimen de Franco, aunque se diese por descontado que sería investigada desde y para sus políticas. En segundo lugar, porque recogió a los profesores y los funcionarios que estaban capacitados para diseñar y analizar encuestas y que hasta el momento no habían tenido un lugar en el que desarrollar sus capacidades. Además el IOP cumplió una función técnica y científica extraordinaria, porque creo la REOP (AHORA REIS) una revista alternativa a la de Balmes, porque hasta ese momento no había nada... La gente que entró en el IOP también lo hizo con mucha fuerza en la docencia universitaria y en política. Muchos de esos profesores procedían de Políticas, y eso tuvo muchísima importancia porque fue lo que facilitó de facto que los estudios políticos se separaran de los económicos y se orientaran a lo sociológico. Las cosas no ocurren porque sí.

(...) La gente nueva que asumiríamos la docencia de la Sociología en la Facultad de Políticas y económicas no teníamos nada que ver con esa tradición Escolástica (del Instituto Balmes). Muchos de ellos llegaron de EE.UU. y desembarcan en masa en el Departamento en que yo estaba, que dirigía el profesor Lisarrague. Cuando él falleció, se convocó la oposición a la Cátedra de sociología, que ganó Salustiano del Campo. Y el profesor del Campo, organizó un Departamento en el que casi toda la gente estaba vinculada al IOP. 
Estas personas ya tenían el soporte académico e institucional para hacer investigación social, hasta donde se podía hacer investigación social en este país. Ese fue el gran cambio, y se produjo entre 1964 y 1968" (entrevista a Manuel Martín Serrano, julio de 2011).

Desde el IOP se comenzaron a desarrollar estudios institucionales, para cuya realización no se recurrió solamente a la creación de una infraestructura de investigación pública e independiente en el seno del IOP, sino que se contó con la colaboración de las empresas de investigación de mercados, que habían comenzado a consolidar su estructura productiva mucho antes de que lo hiciera el sector institucional. De la mano del IOP, las empresas de estudios de consumo entraron de lleno en la producción de estudios de corte sociopolítico y sociológico. Algunos de ellos, como Alef, bajo la dirección técnica de Jesús Ibáñez, siguieron el camino iniciado por DATA con Amando de Miguel y se especializaron precisamente en este tipo de estudios, aunque sin abandonar por ello los tradicionales estudios de consumo.

"En los primeros setenta se hacían muchos estudios sociológicos. No tanto políticos, por motivos obvios, pero sí sociológicos. Hasta casi el final de la década casi no entran estudios verdaderamente de consumo. En los primeros años éramos pocos y más o menos todos hacíamos lo mismo, aunque creo que Alef, por la gente que había allí, tenía un sello definido, una forma de hacer propia que la hacía mejor que otras" (entrevista a José Vicente del Barco, julio de 2011).

“El «Desarrollo» se convierte en seña de identidad y programa político de los nuevos grupos de poder que encabezan los gobiernos franquistas durante la década de los sesenta. Se suceden varios "Planes de desarrollo», que toman su inspiración, pero no su contenido, de los «Planes Quinquenales» de la URSS. De hecho en un contexto internacional que se proponía construir la «Sociedad del bienestar» el régimen franquista alcanza sus mayores tasas de crecimiento económico, y «el desarrollismo» se trasluce en demanda de estudios necesarios para la planificación económica y política. Estudios que inevitablemente tienen que abordar las transformaciones estructurales del país y sus correlativos cambios en los modos de vida, en las formas de ver el mundo y de proyectar el futuro. En mi opinión, ese fue el motivo por el que pocos años después, al inicio de los setenta, los estudios con contenidos referidos a los cambios sociales están entreverados con las investigaciones que encargan las Administraciones Publicas, para diseñar y dar seguimiento a las políticas de desarrollo.

Las instituciones privadas, los profesores e investigadores que al final de los 60 se ocupan de hacer estudios sobre los cambios sociales adquieren peso sociopolítico. Por ejemplo, es lo que ocurre con la FUNDACION FOESSA que financia y da cobertura a los sucesivos «Informes» que dirigieron, entre otros, el profesor Amando de Miguel. Quienes hacen esa clase de estudios son conscientes de que están contribuyendo a la transformación social del país y, por tanto, los temas que eligen y los análisis que hacen están dedicados a las cuestiones que consideraban cruciales para llevar a cabo ese cambio. En los informes de la FUNDACION FOESSA escribe gente muy competente, que expone por primera vez, ahora sí, en términos sociológicos y macro-sociológicos, cómo 
estaba el país a finales de los 60 . Varios de estos investigadores venían de trabajar con Linz. Y ninguno de ellos estaba vinculado a los grupos que entonces se consideraban revolucionarios. Pero en conjunto, dejaron constancia de que la planificación social podía realizarse de un modo diferente, con otras técnicas y distintos objetivos" (entrevista a Manuel Martín Serrano, julio de 2011).

\section{LA CONSOLIDACIÓN DE LA INDUSTRIA DE LA INVESTIGACIÓN}

Este modo de producción de investigación continuó desarrollándose durante los años sesenta y setenta del pasado siglo, consolidando una infraestructura productiva privada dedicada a la producción de investigación, que era utilizada indistintamente para la realización de estudios de consumo, sociopolíticos e institucionales y que, gracias a la difusión de la información que producía en medios públicos y privados, se erigió como la principal fuente de información, contrastada y contrastable, sobre el acontecer social.

Y a partir del momento en que el sector empresarial consolida su posición como fuente de información sobre la realidad social, es cuando adquiere todas las características de una verdadera "Industria de la Investigación", entendida como un sector especializado en la producción de información sobre la realidad social (es decir, en la producción de conocimiento), mediante la utilización de técnicas de investigación social aplicada.

Esta Industria de la Investigación engloba a todo el sector empresarial dedicado a la producción de información en condiciones de mercado, es decir, que realizan investigación por encargo, independientemente de si éste procede de una empresa, de un organismo público o de una institución y sin hacer distinción de los fines -sociales o comerciales- de dicha investigación. Además, su producción de información es un proceso de producción ${ }^{2}$ reglado que se rige por normas y códigos éticos sectoriales internacionales (sancionados desde la asociación profesional), ateniéndose a ciertos "modos de hacer" que se sustentan en la aplicación de técnicas contrastadas de investigación social y que son los únicos reconocidos como válidos por la comunidad investigadora y por sus clientes (quienes encargan la investigación), condicionando el

2 El carácter productivo de la Industria de la Investigación se inscribe dentro de la tendencia de la sociedad capitalista de convertir en proceso productivo cualquier intercambio social, incluidos los que guardan relación con la cultura, como lo es, en este caso, la producción de conocimiento. Según Manuel Martín Serrano (2008), "La sociedad industrial mantuvo la afirmación de que existía una solidaridad armoniosa entre tecnología y cultura. A partir de entonces, esta hipótesis no era un error: constituía una falsificación interesada que excluía de la conciencia de los hombres la cultura no integrada con la forma capitalista de producción. La dominación social se constituyó como dominación cultural; la dominación cultural adquirió la forma de una falsa conciencia de la realidad, que proponía un modelo del mundo en el que la cultura y el sistema de producción estaban reconciliados. El desarrollo histórico de la sociedad industrial ha significado la generalización del valor de cambio como medida universal de los intercambios sociales, tanto a nivel cultural como a nivel productivo. La diferencia que podía existir entre valores materiales y valores culturales ha quedado borrada; el dominio de la producción cultural ha pasado a ser otro ámbito de la producción general". 
funcionamiento global de la industria. Sus principales características como productora de conocimiento pueden resumirse del modo siguiente:

- La investigación se elabora siguiendo un proceso estandarizado: se realiza en condiciones de mercado y está sujeta a un conjunto de normativas y regulaciones propias de la actividad.

- Su proceso de producción utiliza unos dispositivos de captura ideológicamente condicionados, limitados a los procesos y los métodos aceptados por la comunidad investigadora (que están regulados por la normativa sectorial).

- Como en todo proceso de producción, el resultado es un producto manufacturado, constituido por la información que proporciona la investigación: "datos" y "relatos" sobre el acontecer.

- Es elaborado por organizaciones sociales (la Industria de la Investigación) para proporcionar información a otras organizaciones -empresas, instituciones- que encargan la investigación).

- Tiene un valor de uso pre-definido (los objetivos o necesidades de información del cliente que encarga la investigación).

- Dicho producto proporciona una representación ideológica de la realidad. La información sobre los hechos sociales es un "relato" de la realidad, distinto a los hechos que se producen de manera espontánea en dicha realidad.

- Y dicha representación es producida por un sector profesional especializado en interpretar el comportamiento social.

\section{LA INDUSTRIA DE LA INVESTIGACIÓN COMO INSTITUCIÓN MEDIADORA}

La Industria de la Investigación así definida y entendida, adquiere las características de un sector productivo encargado de producir información técnico-científica -es decir, conocimiento-, por lo que puede ser considerada como mediadora social del conocimiento, ya que su producción deviene en relatos de la realidad cuyo objetivo es actuar sobre la interpretación que las personas hacen de ella, tratando de generar consensos en las visiones del mundo, siendo por ello partícipe de las tareas de control social, como modelo de orden ${ }^{3}$.

\footnotetext{
3 "Una de las aplicaciones de la Teoría de la Mediación en el campo de las Ciencias Sociales consiste en el estudio del control social que ejercen las instituciones actuando sobre la interpretación que hacen las personas de la realidad. Participan en esta tarea de control aquellas instituciones sociales que administran la producción y la oferta de información: entre ellas la familia, la escuela, la iglesia, los medios de comunicación de masas. Desde esta perspectiva, son modalidades de control social por el recurso a la
} 
- Está sujeta a unos modos de producción, que incluyen tanto los métodos como los procedimientos que se utilizan (que son los aceptados como válidos por la industria en cada momento) y que son históricamente cambiantes y están sometidos al cambio tecnológico.

- Tanto los modos de producción como los productos finalmente obtenidos, responden a los intereses particulares de quienes encargan la investigación, que pertenecen al rango de los poderes (políticos, económicos y sociales).

Como modelo de orden, la información que produce, en lugar de tratar de comprender la realidad original que investiga, utiliza ésta para ilustrar la forma de consenso (la representación social) correspondiente al modelo del que forma parte. Es decir, el investigador trata de explicar el orden a través de los hechos de esa realidad original ${ }^{4}$.

La producción de conocimiento de esta Industria de la Investigación y, por extensión, de los investigadores que trabajan en ella, cumple un papel mediador que pertenece al ámbito del control social. La reflexividad y la autoconsciencia es una tarea imprescindible para el investigador que quiere alcanzar el nivel epistemológico descrito por Ibáñez ${ }^{5}$ (1985) para no ser "devorado" en el proceso y que impone la necesidad de una permanente vigilancia epistemológica, mediante la reflexión sobre las alteraciones que el proceso de producción de investigación introduce en la realidad social por el mero hecho de estudiarla. En el mismo sentido, Martín Serrano advierte

información todas las acciones que inciden en la enculturización de las personas: estudios reglados: manifestaciones culturales, artísticas, rituales o recreativas: oferta de noticias que circulan por sistemas informales o por los MCM, etc." (Martín Serrano, 2004).

4 "Cuando la mediación introduce un modelo de orden entre las cosas para ofrecer una visión estable del mundo, se produce un cambio importante. La información del mediador cesa de tener por objeto la realidad original, «lo que ocurre». Por el contrario, el mediador, por medio de "lo que ocurre» trata de explicar el orden. El suceso sirve para ilustrar una forma de consenso que ofrece un significado estable para interpretar el mundo. El mediador se sirve del acontecer para reproducir el código" (Martín Serrano, 2008).

5 En su obra Del algoritmo al sujeto. Perspectivas de la investigación social, Jesús Ibáñez hace referencia a la necesidad de reflexión del investigador sobre su propia participación en el proceso de investigación como único modo de alcanzar el nivel epistemológico. "El sociólogo debe situarse en un nivel muy alto de reflexividad: Un nivel cero sería el nivel de percepción ingenua de los hechos: nivel del técnico investigador que percibe los hechos como «data» y no como "capta». Un nivel uno sería el nivel de reflexión sobre los dispositivos de captura de los hechos: nivel de tecnólogo que produce efectos de verdad en una dirección operatoria. Un nivel dos sería el de la re-reflexión sobre los dispositivos de integración teórica en el que se almacenan los datos captados: nivel del metodólogo que produce efectos de verdad en una dirección sistémica. Un nivel tres sería el nivel de re-rereflexión sobre los dispositivos de integración de los dispositivos de captura y de los dispositivos de construcción: sería el nivel del epistemólogo, que ya no produce efectos de verdad, sino efectos de supervivencia; que no se sitúa en la perspectiva semántica o de significación, sino en la pragmática o de sentido" (Ibáñez, 1985). 
de la imposibilidad de mantener la neutralidad frente al objeto de estudio, cuando esta es interpretada a través de un modelo de orden ${ }^{6}$.

Como ya se ha mencionado en la definición y caracterización de la Industria de la Investigación, entendemos que se trata de una institución mediadora del conocimiento, en el sentido en que Martín Serrano concibe la mediación:

a) En cuanto que productora de un conocimiento ideológicamente condicionado, es una institución normativa (busca la concordancia entre conductas y normas), lo que la hace apta para ser estudiada según la teoría de la mediación ${ }^{7}$.

b) En su modelo de mediación, los fenómenos sociales (hábitos, actitudes, percepciones, etc. de determinados grupos sociales) son los objetos del medio humano que la Industria de la Investigación, a través de un proceso de producción de conocimiento (proceso cognitivo) pone en relación con los objetivos de su cliente, ofreciendo como resultado final un producto que es una interpretación ideológica de la realidad.

c) El condicionamiento ideológico al que está sometida la industria, tanto por la propia regulación sectorial (lo aceptado como "buena praxis") como por estar sujeta a los intereses particulares de sus clientes, garantiza que dicho producto responda a un determinado modelo de orden, que es el que pone los límites al repertorio de significaciones posibles de la información contenida en dicho producto ${ }^{8}$.

Para ejercer su papel mediador, el producto de la Industria de la Investigación tiene que utilizar un determinado "código", que tiene que ser compartido a priori entre el mediador (el investigador) y los receptores (los clientes y, a través de la acción de estos, la sociedad en general).

6 "La relación del conocimiento con la realidad social podría calificarse como el gran estupor; asombro que surge de lo indefinido, es decir, de la infinitud de las definiciones posibles que cabe atribuir al universo de los estímulos simultáneamente presentes. Cuando el mediador introduce un modelo de orden, los estímulos se transforman en «datos». La relación directa del hombre cara a los «estímulos» es la contemplación mística: incluso la distinción entre sujeto y objeto desaparece. La relación del hombre cara a los «datos» es la clasificación lógica. En este último caso desaparece el estupor al mismo tiempo que la neutralidad del sujeto respecto a los objetos" (Martín Serrano, 2008).

7 "Todas las instituciones normativas podrían ser estudiadas desde el punto de vista de la teoría de la mediación, en cuanto mediadoras en los procesos sociales" (Martín Serrano, 2008: 50).

8 "Definimos la mediación como la actividad de control social que impone límites a lo que podría ser dicho, y a las maneras de decirlo, por medio de un sistema de orden (...) Se puede hablar de mediación cuando ciertos objetos del medio humano (materiales o inmateriales) van a ser relacionados con ciertos objetivos, a través de un proceso de interpretación de la realidad que dirige los comportamientos y las acciones, mediando siempre un proceso cognitivo" (Martín Serrano, 2008). 
Y siguiendo a Martín Serrano, dicho "código" está implícito en el modelo de mediación de la industria, en un doble sentido (Martín Serrano, 2008):

- Como estructura: la producción de investigación puede entenderse como un sistema codificante destinado a uniformar la diversidad del acontecer desde un punto de vista sociocultural. Código y estructura se identifican en significado.

- Como coerción: el producto de la investigación contiene criterios de verdad (el conocimiento) "que sirven para corregir las desviaciones del conocimiento y del comportamiento respecto a un único propósito prefijado". Según Durkheim, este tipo de código forma parte de un Modelo de la coerción, que toma partido acerca de "lo que ocurre". En este contexto, "código" equivale a ideología.

\section{CONCLUSIÓN: EL PAPEL INSTRUMENTAL DE LA INDUSTRIA DE LA INVESTIGACIÓN EN LA REPRO- DUCCIÓN Y EL CONTROL SOCIAL}

La industria de la Investigación tiene, por tanto, un carácter instrumental como investigación social aplicada (es decir, que persigue un fin práctico) cuyo fin es la acción social. Y este carácter instrumental se infiere también al producto elaborado por la industria: el producto (informe o memoria de resultados) es un conjunto de informaciones, organizadas como un relato que contiene una representación de la realidad (lo que los sujetos sociales estudiados -consumidores, ciudadanos, votantes- hacen, piensan u opinan) y que se elabora con un objetivo predefinido por otros sujetos sociales (quienes encargan la investigación), cuyo fin último (si asumimos su carácter instrumental) consiste en lograr una acción que cambie o reproduzca un comportamiento (la forma de consumir, pensar, votar o actuar).

Es decir, la Industria de la Investigación persigue una producción de información necesaria para elaborar un tipo de relatos que puedan ser aceptados tanto por sus destinatarios inmediatos (la empresa, organismo o institución que los encarga) como por los grupos sociales sobre los que estos pretenden actuar (los consumidores, los ciudadanos, los votantes...) y, para ello, creemos que debe cumplir los siguientes requisitos, fijados a partir de los expuestos por Martín Serrano en La producción social de comunicación:

a) El conocimiento producido por la Industria de la Investigación debe proporcionar una explicación del fenómeno social estudiado capaz de armonizar el cambio y la transformación pretendidos, con el mantenimiento de la organización social y de las instituciones promotoras de la investigación. La explicación debe incluir un modelo de comportamiento para todos los sujetos sociales involucrados: el colectivo estudiado y el cliente que encarga la investigación, aunque las opciones para uno y otro sean diferentes e incluso contrapuestas. 
b) Debe proporcionar gratificaciones cognitivas y afectivas a dichos sujetos, desde un plano subjetivo, para poder ser asumida socialmente como una interpretación válida del mundo. Para que se produzca esta interiorización, el conocimiento ideológico tiene que satisfacer necesidades esenciales de los sujetos, como mínimo las que se refieren a la conciencia de pertenencia a un grupo y al sentimiento de seguridad.

Es en este contexto en el que la actividad de la Industria de la Investigación puede -y debe- ser considerada como una producción social de conocimiento mediado ${ }^{9}$, en el sentido propuesto por Martín Serrano.

\section{BIBLIOGRAFÍA}

HERNÁNDEZ FERNÁNDEZ, C. (2013): La producción de conocimiento sociológico en España. Madrid: Universidad Complutese de Madrid. Tesis doctoral. Disponible en http://eprints.ucm.es/20054/.

IBÁÑEZ, J. (1985): Del algoritmo al sujeto. Perspectivas de la investigación social. Madrid: Siglo $X X I$.

${ }^{9}$ Esta definición se ha elaborado a partir de la que hace Martín Serrano en La producción social de comunicación, cuando la describe como una "actividad comunicativa que produce una información que está destinada al conjunto de los miembros de una comunidad y que se refiere a aquellos aconteceres que afectan o pueden afectar a la existencia del grupo como tal grupo (...) la dimensión cognitiva del producto comunicativo se manifiesta porque está expresamente confeccionado para ofrecer información. El resultado es un 'relato'. El relato que trata del entorno es la manifestación objetivada de una representación del acontecer" (Martín Serrano, 2004: 76).

Asimismo, toma como referencia su descripción de las representaciones ideológicas, en la misma obra: "Las representaciones ideológicas capaces de mantener el interés del conjunto de la sociedad en la perpetuación de ese orden social tienen que proveer de algo más que propaganda política. Cuando una representación de la realidad sirve para el control social puede asegurarse que esa representación satisface dos necesidades: a) Proporciona una teoría de la sociedad en la cual se armonizan la transformación de las formas de vida y de los valores, con el mantenimiento de esa organización social y de sus instituciones. En dicha teoría debe de haber un modelo de comportamiento no sólo para los miembros de las clases dependientes, sino también para quienes forman parte de las clases dominantes, aunque uno y otro contengan opciones diferentes. Por esa razón las representaciones ideológicas de la realidad pueden ser partidistas pero nunca cortas de miras. b) Proporciona a nivel subjetivo gratificaciones cognitivas y afectivas. Supuesta la existencia de una teoría ideológica. Su eficacia para el control social depende de que sea asumida por los miembros de esa sociedad como una interpretación válida del mundo. Para que se produzca esta interiorización, la teoría ideológica tiene que satisfacer necesidades esenciales de la personalidad: al menos aquellas que se refieren a la conciencia de pertenencia a un grupo y al sentimiento de seguridad. Por eso las representaciones ideológicas de la realidad pueden ser falsas, pero nunca banales" (Martín Serrano, 2004: 43). 
LAMO DE ESPINOSA, E. (1992): "La sociologia española desde 1939", Theoria. Proyecto Crítico de Ciencias Sociales - Eurotheo. La sociología en la Unión Europea. España. Disponible en http://goo.gl/Qrk7RE. Consultado el 7 de mayo de 2012.

MARTÍN SERRANO, M. (1978): "Bases para una epistemología general de las ciencias sociales", REIS, n은, pp. 17-55.

MARTÍN SERRANO, M. (1992): "Las relaciones macrosociológicas entre acción y comunicación", en Moya, C., Pérez-Agote, A., Salcedo, J. y Tezanos, J. F. (coords.): Escritos de teoría sociológica en homenaje a Luís Rodríguez Zúñiga. Madrid: Centro de Investigaciones Sociológicas, pp. 671-682.

MARTÍN SERRANO, M. (2004): La producción social de comunicación. Madrid: Alianza. 3a edición.

MARTíN SERRANO, M.( 2006a): "La vocación de la sociología académica española”, RES, no 6, pp. 107-113.

MARTÍN SERRANO, M. (2006b): "Para qué sirve estudiar teoría de la comunicación", Contratexto, no 4, no 14, pp. 41-48.

MARTÍN SERRANO, M. (2007): Teoría de la comunicación. Madrid: McGraw-Hill.

MARTíN SERRANO, M. (2008): La mediación social. Edición conmemorativa del 30 aniversario. Madrid: Akal.

ORTí, A. (2007): "Veinticinco años después: el oficio de sociólogo en la España plural”, RES, no 7, pp. 27-75.

Coral HeRnández Fernández es Profesora Asociada del Departamento de Sociología IV de la Facultad de Ciencias de la Información de la Universidad Complutense de Madrid (UCM). Licenciada y Doctora en Sociología por la UCM (2012). Previamente ha sido docente e investigadora de la Universidad Nebrija (2008-2012). Dedicada a la investigación de mercados, a los estudios de comunicación y al conocimiento del consumidor desde hace más de 30 años, cuenta con amplia experiencia en la utilización de todo tipo de técnicas y métodos de investigación, tanto cualitativas como cuantitativas y tanto de corte clásico (segmentación, análisis multivariante, etc.) como de reciente desarrollo (técnicas online, medición y analítica web, análisis de contenidos semánticos, etc.). Actualmente es consultora independiente en investigación de mercados, bajo la marca CHF RESEARCH y Head of Consumer Insights en Smartme Group. Es miembro de la organización profesional AEDEMO y ha suscrito el código ético ICC-ESOMAR. Correo electrónico: chernandezf@pdi.ucm.es. 